\title{
Sparse Signal Representations of Bearing Fault Signals for Exhibiting Bearing Fault Features
}

\author{
Wei Peng, ${ }^{1,2}$ Dong Wang, ${ }^{3}$ Changqing Shen, ${ }^{4}$ and Dongni Liu ${ }^{3}$ \\ ${ }^{1}$ Zhongshan Institute, University of Electronic Science and Technology of China, Zhongshan 528402, China \\ ${ }^{2}$ Institute of Reliability Engineering, School of Mechatronics Engineering, University of Electronic Science and Technology of China, \\ Chengdu 610051, China \\ ${ }^{3}$ Department of Systems Engineering and Engineering Management, City University of Hong Kong, Tat Chee Avenue, \\ Kowloon, Hong Kong \\ ${ }^{4}$ School of Mechanical and Electrical Engineering, Soochow University, Suzhou 215021, China \\ Correspondence should be addressed to Dong Wang; dongwang4-c@my.cityu.edu.hk
}

Received 5 May 2015; Revised 7 October 2015; Accepted 12 October 2015

Academic Editor: Peng Chen

Copyright @ 2016 Wei Peng et al. This is an open access article distributed under the Creative Commons Attribution License, which permits unrestricted use, distribution, and reproduction in any medium, provided the original work is properly cited.

\begin{abstract}
Sparse signal representations attract much attention in the community of signal processing because only a few coefficients are required to represent a signal and these coefficients make the signal understandable. For bearing faults' diagnosis, bearing faults signals collected from transducers are often overwhelmed by strong low-frequency periodic signals and heavy noises. In this paper, a joint signal processing method is proposed to extract sparse envelope coefficients, which are the sparse signal representations of bearing fault signals. Firstly, to enhance bearing fault signals, particle swarm optimization is introduced to tune the parameters of wavelet transform and the optimal wavelet transform is used for retaining one of the resonant frequency bands. Thus, sparse wavelet coefficients are obtained. Secondly, to reduce the in-band noises existing in the sparse wavelet coefficients, an adaptive morphological analysis with an iterative local maximum detection method is developed to extract sparse envelope coefficients. Simulated and real bearing fault signals are investigated to illustrate how the sparse envelope coefficients are extracted. The results show that the sparse envelope coefficients can be used to represent bearing fault features and identify different localized bearing faults.
\end{abstract}

\section{Introduction}

Rolling element bearings are commonly used in machines to support rotation shafts. Their failures may cause unexpected machine breakdown and lead to huge economic loss. A rolling element bearing consists of an outer race, an inner race, several rollers, and a cage. Once a defect is formed on the surface of either the outer race or the inner race, an impact is generated by each of the rollers striking the defect surface and thus it excites the resonant frequencies of the structures between bearings and transducers [1-4]. Therefore, to extract bearing fault features, envelope analysis is one of the most effective methods. To conduct envelope analysis, two steps are needed. The first step aims to use a band-pass filter to retain one of the resonant frequency bands for enhancing the signal to noise ratio of bearing fault signals because bearing fault signals are often overwhelmed by strong low-frequency vibration components and heavy noises. The second step is extracting the envelope of the signals filtered by the bandpass filter [5-7]. Moreover, if the envelope signals can be represented by a few coefficients, namely, sparse envelope coefficients [8], bearing fault signals are more understandable and easily interpreted.

In this paper, a joint signal processing method for extraction of sparse envelope coefficients is proposed. Firstly, an optimal wavelet filter is tuned by particle swarm optimization (PSO). For the use of wavelet transform, the similarity between a signal and a wavelet is the most concerned and the high similarity can result in large wavelet coefficients so as to highlight hidden transients. Because the shape of a Morlet wavelet is similar with the transients caused by localized bearing faults and the Morlet wavelet has a band-pass property, 
which can be used to retain one of the resonant frequency bands and enhance the signal to noise ratio of bearing fault signals, the Morlet wavelet is chosen in this paper [9-14]. To automatically tune the parameters of the Morlet wavelet, two aspects including a metric and an optimization algorithm must be determined. In the past years, some metrics, such as kurtosis, entropy, smoothness index, and sparsity measurement, have been investigated for optimization of the Morlet wavelet transform [9-14]. Their comparisons show that the sparsity measurement can generate better visual inspection performance and highlight bearing fault signatures, such as bearing fault characteristic frequency and its harmonics [15]. Therefore, the metric used in this paper is the sparsity measurement. To achieve the global optimal parameters of the Morlet wavelet transform, genetic algorithm, differential evolution, and stepwise scanning have been studied [9-15]. To explore the feasibility of an easy and simple optimization algorithm, particle swarm optimization is used in this paper to tune the sparsity measurement because the core of particle swarm optimization is based on the simple physical relationship among position, velocity, and acceleration. The use of PSO is simply introduced as follows. A number of particles move in a searching space. Then, a simple mathematic algorithm searches the best position by sharing the cognitive and social influences among all particles in the searching space [16-18]. For intelligent machine fault diagnosis, PSO was used to tune the parameters of support vector machine, artificial neural network, and proximal support vector machine, respectively [19-21]. The results show that these statistical prediction models combined with PSO have good prediction accuracies for identification of different machine faults.

Secondly, even though the optimal wavelet filtering is conducted on bearing fault signals for enhancement of bearing fault signatures, in-band noises still exist because the optimal wavelet filtering cannot remove the noises existing in the retained resonant frequency band. In recent years, an attracting method called morphological analysis (MA) is widely investigated due to its simplicity and effectiveness in extracting envelope signals [22-29]. MA aims to use Minkowski addition and subtraction to intersect the morphological features of bearing fault signals with a structuring element (SE). However, if the morphological features are overwhelmed by other strong vibration components and heavy noises, MA may fail to retain the morphological features of bearing fault signals. Therefore, MA can be used to postprocess bearing fault signals, if the signal to noise ratio of bearing fault signals is not high. In this paper, an adaptive MA with an iterative local maximum detection method is developed to automatically find the optimal parameter of MA and extract sparse envelope signals so as to exhibit bearing fault features.

The rest of this paper is outlined as follows. Section 2 introduces the fundamental algorithms used in this paper. These algorithms include wavelet transform, particle swarm optimization, and morphological analysis. In Section 3, extraction of sparse envelope coefficients from bearing fault signals is proposed. In Section 4, simulated and real bearing faults signals are analyzed by using the proposed method. Conclusions are drawn in Section 5.

\section{Fundamental Algorithms}

2.1. Fundamental Theory of Wavelet Transform. Wavelet transform aims to calculate the inner product between an artificial wavelet and a signal. The mathematical formula for wavelet transform is defined as follows [30, 31]:

$$
\begin{aligned}
W f(u, s) & =\int_{-\infty}^{+\infty} f(t) \times \frac{1}{\sqrt{s}} \overline{\psi\left(\frac{u-t}{s}\right)} d t \\
& =f(u) * \psi_{s}^{\prime}(u), \\
\psi_{s}^{\prime}(t) & =\overline{\frac{1}{\sqrt{s}} \psi\left(\frac{-t}{s}\right)},
\end{aligned}
$$

where $s$ is the scale parameter and $u$ is the translation parameter. $*$ represents the convolution operator. - takes the complex conjugate of the signal. According to the properties of Fourier transform, (1) is rewritten as

$$
W f(u, s)=f(u) * \psi_{s}^{\prime}(u)=F^{-1}\left(\widehat{f}(f) \times \sqrt{s} \widehat{\psi_{s}^{\prime}}(s f)\right),
$$

where $F^{-1}$ is the inverse Fourier transform and $\wedge$ is the Fourier transform. As explained in Introduction, the complex Morlet wavelet is chosen in this paper. Its temporal waveform and the corresponding frequency spectrum are given as follows:

$$
\begin{gathered}
\psi(t)=\frac{\sigma}{\sqrt{\pi}} e^{-\sigma^{2} t^{2}} e^{j 2 \pi f_{c} t}, \\
\vartheta(f)=e^{-\pi^{2} / \sigma^{2} \times\left(f-f_{c}\right)^{2}} .
\end{gathered}
$$

From (5), it is obvious that the complex Morlet wavelet has a band-pass property and its frequency support is constrained to the frequency band $\left[f_{c}-\sigma / 2, f_{c}+\sigma / 2\right]$. Because any wavelet must satisfy the admission condition, which means that the integration of a wavelet over time must be equal to zero, the following equation should be satisfied:

$$
\vartheta(0)=e^{-\pi^{2} / \sigma^{2} \times\left(0-f_{c}\right)^{2}}=0 .
$$

It is not difficult to verify that if $f_{c} / \sigma>1.3, \vartheta(0) \approx 0$.

\subsection{Fundamental Theory of Particle Swarm Optimization.} PSO is a population based stochastic optimization method, which optimizes a metric by iteratively moving a number of particles in a searching space, according to some simple mathematical formulas related to the positions and velocities of all particles. Each particle represents one potential solution to the optimization problem. The movements of the particles are guided by their local best positions and the best swarm position. The basic theory of PSO is described in the following [16]. Considering the physical relationship among position, velocity, and acceleration, the following basic physic principle is listed as follows:

$$
x_{i}(k+1)=x_{i}(k)+v_{i}(k)+\frac{1}{2} a_{i}(k),
$$


where $x_{i}(k+1)$ and $x_{i}(k)$ mean the $k+1$ th and the $k$ th positions of the $i$ th particle, respectively. $v_{i}(k)$ is the $k$ th velocity of the $i$ th particle. $a_{i}(k)$ is the $k$ th acceleration of the $i$ th particle. Then, the acceleration of the $i$ th particle is divided into a cognitive acceleration, which is proportional to the distance between the current position of the $i$ th particle and the personal best position $p_{i}(k)$ of the $i$ th particle, and a social acceleration, which is proportional to the distance between the current position of the $i$ th particle and the global best position $g(k)$ of the $i$ th particle. To make these two new parts more flexible, a cognitive coefficient $c_{1}$ and a social coefficient $c_{2}$ are used. Consequently, (7) is reformulated as

$$
\begin{aligned}
x_{i}(k+1)= & x_{i}(k)+v_{i}(k)+\frac{1}{2} c_{1}\left(p_{i}(k)-x_{i}(k)\right) \\
& +\frac{1}{2} c_{2}\left(g(k)-x_{i}(k)\right) .
\end{aligned}
$$

Then, in order to prevent the velocities from getting out of control, the influence of friction is considered by introducing an inertia weight $\omega$, which is smaller than 1 , to (8). Besides, the constant $1 / 2$ is replaced by two random numbers $r_{1 i}$ and $r_{2 i}$, which are limited to the values between 0 and 1 . Equation $(8)$ is revised as follows:

$$
\begin{aligned}
x_{i}(k+1)= & x_{i}(k)+\omega v_{i}(k)+r_{1 i} c_{1}\left(p_{i}(k)-x_{i}(k)\right) \\
& +r_{2 i}(k) c_{2}\left(g(k)-x_{i}(k)\right) .
\end{aligned}
$$

At last, (9) consists of the following velocity and position update equations:

$$
\begin{aligned}
v_{i}(k+1)= & \omega v_{i}(k)+r_{1 i} c_{1}\left(p_{i}(k)-x_{i}(k)\right) \\
& +r_{2 i}(k) c_{2}\left(g(k)-x_{i}(k)\right), \\
x_{i}(k+1)= & x_{i}(k)+v_{i}(k+1) .
\end{aligned}
$$

2.3. Fundamental Theory of Morphological Analysis. MA aims to extract the morphological shape of a temporal signal. It uses a structuring element to intersect with the temporal signal. Let $f(n)$ be the one-dimensional signal over a domain $F=(0,1,2, \ldots, N-1)$ and let $g(n)$ be the structuring element over a domain $G=(0,1,2, \ldots, M-1)$. The basic morphology operators include the dilation operator and the erosion operator, which are related to Minkowski addition and subtraction. The equations for the erosion operator $\ominus$ and the dilation operator $\oplus$ are defined as follows [22]:

$$
\begin{aligned}
(f \ominus g)(n)=\min [f(n+m)-g(m)], & \\
& m \in 0,1,2, \ldots, M-1, \\
(f \oplus g)(n)=\max [f(n-m)+g(m)], & \\
& m \in 0,1,2, \ldots, M-1 .
\end{aligned}
$$

The erosion operator reduces the wave peaks and enlarges the signal minima. On the contrary, the dilation operator increases the wave valleys and enlarges signal maxima [24]. Other morphological operators are constructed based on the combination of the above two operators. Some popular morphological operators used in bearing fault diagnosis are introduced in the following. The opening operator and the closing operator are defined as:

$$
\begin{aligned}
& (f \circ g)(n)=\left[\left(f \ominus g^{s}\right) \oplus g\right](n), \\
& (f \circ g)(n)=\left[\left(f \oplus g^{s}\right) \ominus g\right](n),
\end{aligned}
$$

where $g^{s}(n)$ is the reflection of $g(n)$. The opening operator function is smoothing the signal from the bottom by cutting wave peaks and the closing operator function is smoothing the signal from the top by filling up its wave valleys [24]. The average operator (AVG), the difference operator (DIF), the Black Top-Hat operator (BTH), and the White Top-Hat operator (WTH) are defined as [32]

$$
\begin{aligned}
\operatorname{AVG}(f) & =\frac{(f \cdot g+f \circ g)}{2}, \\
\operatorname{DIF}(f) & =f \cdot g-f \circ g, \\
\operatorname{BTH}(f) & =f \cdot g-f, \\
\operatorname{WTH}(f) & =f-f \circ g .
\end{aligned}
$$

The ability of the average operator lies in flattening both the positive and negative impulsive features. On the contrary, the difference operator is used to extract both the positive and negative impulsive features. The Black Top-Hat and the White Top-Hat operators are employed to extract the negative and positive impulsive features, respectively.

\section{Extraction of Sparse Envelope Coefficients for Exhibiting Bearing Fault Features}

It is not difficult to find that the morphological features extracted by using MA fully depend on the shape of a temporal signal. Because of the interruption from strong low-frequency periodic components and heavy noises, the morphological features of bearing fault signals are prone to be overwhelmed. Therefore, it is necessary to enhance weak bearing fault signals prior to the use of MA. As illustrated in the previous sections, the complex Morlet wavelet optimized by PSO is used to preprocess bearing fault signals and an adaptive MA is developed to postprocess bearing fault signals and to extract sparse envelope coefficients for exhibiting bearing fault features. The flowchart of the proposed method is shown in Figure 1. Each step used in Figure 1 is detailed in the following paragraphs.

Step 1. Load an original bearing fault vibration signal, which is collected by using a transducer attached to a bearing housing.

Step 2. The parameters of the complex Morlet wavelet are tuned by PSO. First, the parameters of particle swarm optimization must be initialized. Each particle corresponds to two-dimensional coordinates, which are used to represent the center frequency and bandwidth of the complex Morlet 


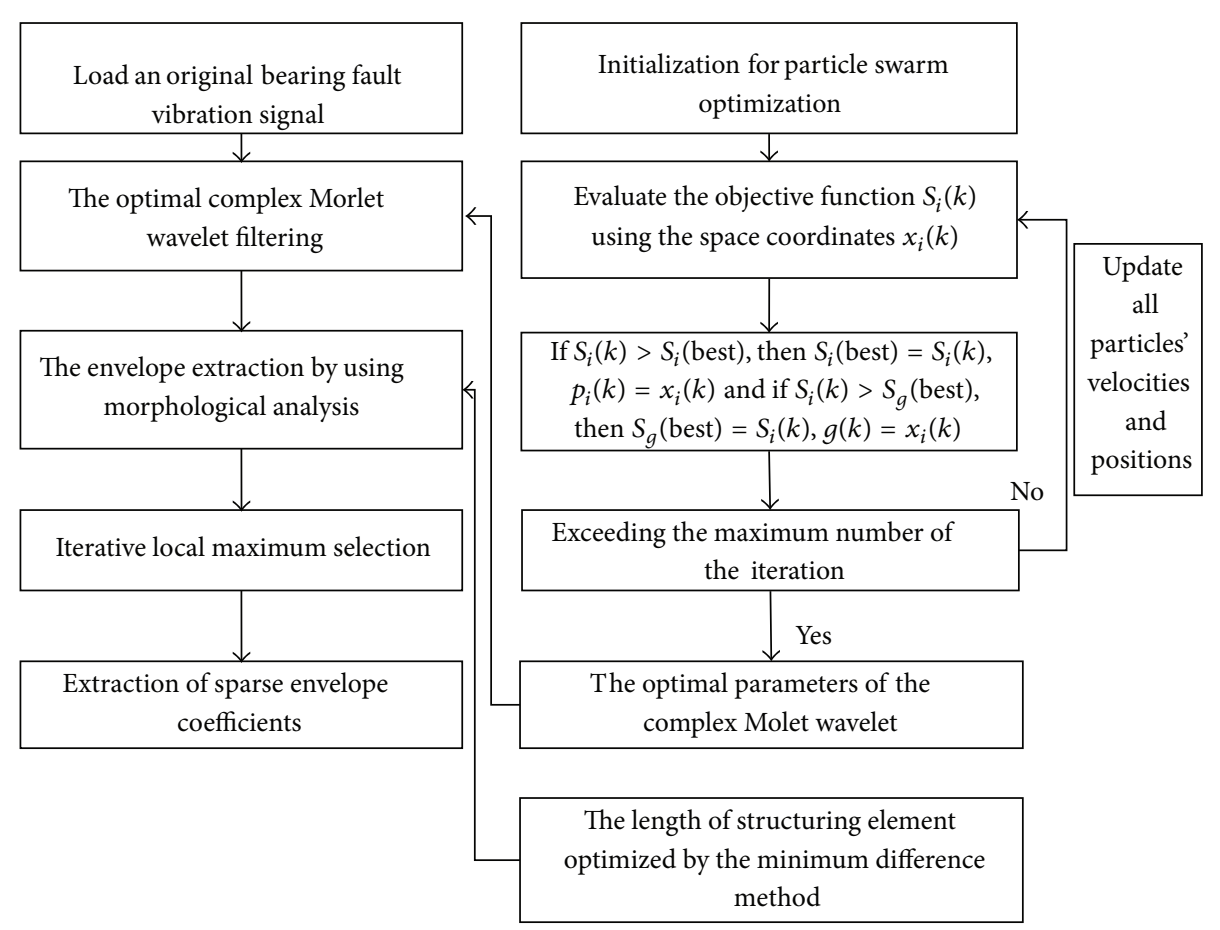

FIGURE 1: The flowchart of the developed method for extracting sparse envelope coefficients.

wavelet, respectively. The searching range for the center frequency was set to the frequency band ranging from $0.1 \times F_{s}$ to $0.4 \times F_{s}$ for avoiding the interruption caused by the lowfrequency vibration components. The searching range for the bandwidth is from $3 \times F_{I}$ to $0.2 \times F_{s}$. Here, to contain enough fault signatures, the minimum bandwidth should be three times larger than the inner race fault characteristic frequency. $F_{s}$ is the sampling frequency and $F_{I}$ is the inner race fault characteristic frequency, which will be formulated later. According to the literature review $[17,18]$, the inertia weight, the number of particles, and the maximum iteration were empirically set to $0.9,24$, and 50 , respectively, because these parameters are sufficiently large for solving an optimization problem. For all particles, the initial positions are randomly generated within the specified searching ranges. The metric, namely, sparsity measurement, is defined as [15]

$$
\begin{aligned}
& S_{i}(k)=\frac{\sqrt{\sum_{n=1}^{L} b_{i}^{k}(n)^{2}}}{\sum_{n=1}^{L}\left|b_{i}^{k}(n)\right|}=\frac{\left\|b_{i}^{k}(n)\right\|_{2}}{\left\|b_{i}^{k}(n)\right\|_{1}} \\
& \quad 1 \leq i \leq 24,1 \leq k \leq 50,
\end{aligned}
$$

where $\left\|b_{i}^{k}(n)\right\|_{2}$ and $\left\|b_{i}^{k}(n)\right\|_{1}$ are $L_{2}$ norm and $L_{1}$ norm, respectively. $b_{i}^{k}(n)$ represents the signal filtered by the complex Morlet wavelet transform, the parameters of which are the two-dimensional coordinates $x_{i}(k)$ of the $i$ th particle at the $k$ th iteration. $L$ is the length of the signal. At the $k$ th iteration, if the $S_{i}(k)$ of the $i$ th particle is larger than the personal largest sparsity value $S_{p}$ (best) of the $i$ th particle (recorded before the $k$ th iteration), the personal best position of the $i$ th particle is updated by its current position $x_{i}(k)$.
Then, if the $S_{i}(k)$ of the $i$ th particle is larger than the global largest sparsity value $S_{g}$ (best) of all particles (recorded before the $k$ th iteration), the global best position of all particles is updated by the current position $x_{i}(k)$ of the $i$ th particle. At last, repeat the above steps until the maximum iteration is reached. The optimal parameters of the complex Morlet wavelet are automatically established by PSO with the maximum sparsity. Here, the real part of the filtered signal obtained by the optimal complex Morlet wavelet is denoted by $b_{\text {opt }}^{\text {real }}(n)$.

Step 3. Once the weak bearing fault signal is enhanced by the optimal complex Morlet wavelet, MA is performed to get sparse envelope coefficients. As introduced in Section 2.3, there are some available morphological operators. To select a proper morphological operator, their comparisons for processing a simulated signal are shown in Figure 2. The structuring element is the flat element with a length of 30 samples because the flat element is a simple and effective element to process a one-dimensional signal [22, 24, 27, 28]. From the results shown in Figure 2, the closing and opening operators can be used to extract the positive and negative envelopes of the signal, respectively. In this paper, only the closing operator is used because the positive envelope is used for further analyses. After both morphological operator and the structuring element are determined, it is necessary to determine the length of the flat element to extract the morphological features of bearing fault signals and suppress in-band noises.

Reference [22] reported a method to empirically decide the length of the flat element and indicated that the optimal 


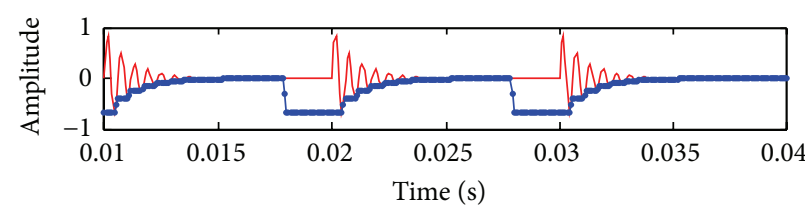

(a)

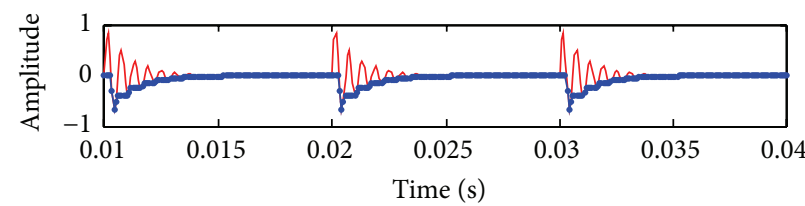

(c)

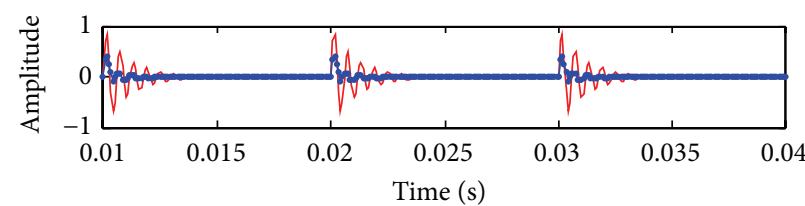

(e)

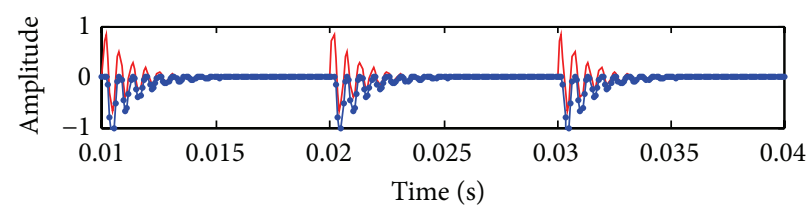

(g)

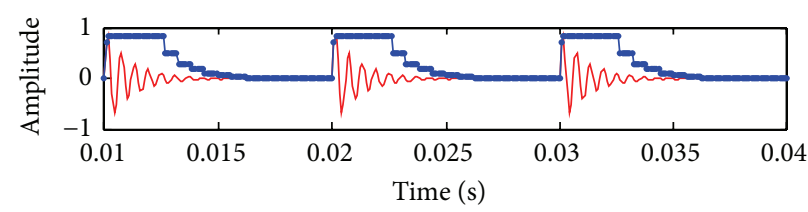

(b)

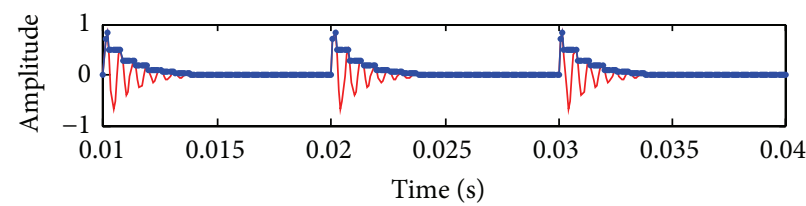

(d)

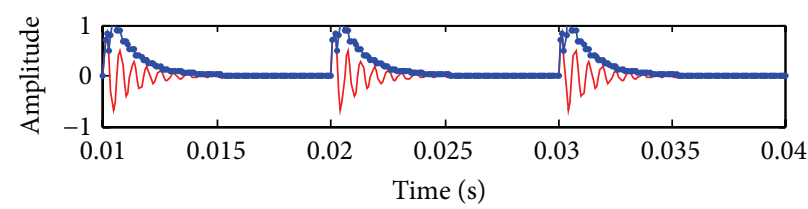

(f)

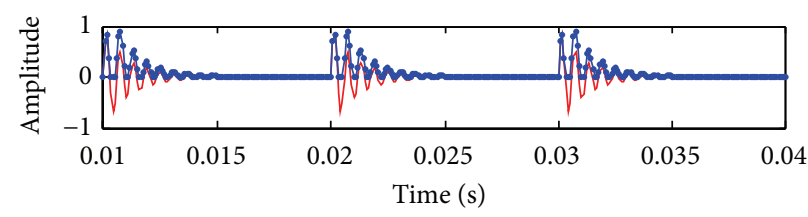

(h)

FIGURE 2: The signals obtained by using various morphological operators: (a) the signal filtered by the erosion operator; (b) the signal filtered by the dilation operator; (c) the signal filtered by opening operator; (d) the signal filtered by the closing operator; (e) the signal filtered by the AVG operator; (f) the signal filtered by the DIF operator; (g) the signal filtered by the BTH operator; and (h) the signal filtered by the WTH operator.

length should be 0.6 to 0.7 of the bearing fault period. However, in our previous research [26], it is found that the empirical optimal length is not always effective for extracting bearing fault features. This paper develops an adaptive morphological analysis with an iterative local maximum detection method to automatically retain sparse envelope coefficients. $b_{\mathrm{opt}}^{\text {real }}(n)$ is processed by the closing operator with various flat element lengths and their corresponding results are denoted by $m m_{\mathrm{opt}}^{\text {real }}(n, l)$, where $l$ is the length of the flat element used in the closing operator. The maximum length of the flat element does not exceed the desired bearing fault period $T$ (unit: samples), which will be defined later. First, find the local maxima $\operatorname{lm}(j), j=1,2, \ldots, N$ of the signal $m m_{\text {opt }}^{\text {real }}(n, l)$ and calculate its local maxima number $N$. Subtract the theoretical impulsive number $Z$ from the local maxima number $N$ and obtain a difference (considering the influence of the negative difference, the absolute value of the difference is used in this paper). The theoretical impulsive number can be calculated as

$$
Z=\operatorname{round}\left(\frac{L}{T}\right),
$$

where the round $(*)$ function is taking the element to the nearest integer.
The bearing fault period $T$ can be represented by the outer race fault characteristic period $T_{o}$, the inner race fault characteristic period $T_{I}$, and rolling element fault characteristic period $T_{B}$. The calculations of these periods are given by

$$
\begin{aligned}
& T_{o}=\frac{1}{\left(\left(P \times f_{s}\right) / 2\right)(1-(d / D) \cos \alpha)^{\prime}}, \\
& T_{I}=\frac{1}{\left(\left(P \times f_{s}\right) / 2\right)(1+(d / D) \cos \alpha)^{\prime}}, \\
& T_{B}=\frac{1}{\left(\left(D \times f_{s}\right) / d\right)\left(1-\left(d^{2} / D^{2}\right) \cos \alpha\right)^{\prime}},
\end{aligned}
$$

where $f_{s}$ is the shaft rotation frequency in Hz. $d$ and $D$ are diameters of the rolling element and the pitch, respectively. $P$ is the number of rolling elements and $\alpha$ is the contact angle.

If the difference between the local maxima number $N$ and the theoretical impulsive number $Z$ is the smallest, it means that the extracted signal by using the closing operator is the best one because the signal filtered by the complex Morlet transform has a high signal to noise ratio and then the local maxima of the filtered signal are the most possible to be the local peaks of the impulses caused by localized bearing faults. Therefore, the optimal length of the flat element can be decided. However, it should be pointed out that sometimes 
the amplitudes of random noises may affect the number of local maxima. Therefore, the iterative local maximum selection is developed to remove the pseudo- and abnormal locations of the local maxima.

Locate all local maxima and denote them by $\operatorname{llm}(j), j=$ $1,2, \ldots, N$. Then, calculate the adjacent distances between two successive locations $\operatorname{llm}(j+1)-\operatorname{llm}(j)$. There are $N-1$ $\operatorname{distances} \operatorname{dis}(j), j=1,2, \ldots, N-1$.

Assume that all the calculated distances are subject to a normal distribution $N\left(\mu, o^{2}\right)$. Here, $\mu$ and $o^{2}$ are the mean and the variance, respectively. In statistics and probability theory, standard deviation $o$ can be used to measure the diversity of samples. It is expected that all distances $\operatorname{dis}(j)$, $j=1,2, \ldots, N-1$ tend to be close to the mean and have a low standard deviation. Therefore, a high standard deviation can be employed to reject the outlier (abnormal distance). In this paper, two standard deviations $2 o$ are used. Then, it is believed that about the 95 percent of the all distances are within two standard deviations $(\mu \pm 2 o)$. Moreover, only distances that are smaller than $(\mu-2 o)$ are rejected because all distances are always positive.

Find the abnormal distances, which are denoted by $\operatorname{llm}(z+1)-\operatorname{llm}(z)$. Calculate the distances $\operatorname{llm}(z+2)-\operatorname{llm}(z+1)$ and $\operatorname{llm}(z)-\operatorname{llm}(z-1)$, respectively. If $\operatorname{llm}(z+2)-\operatorname{llm}(z+1)>$ $\operatorname{llm}(z)-\operatorname{llm}(z-1), \operatorname{llm}(z)$ is discarded. Otherwise, $\operatorname{llm}(z+1)$ is discarded.

Repeat the iterative local maximum selection method until all distances are within two standard deviations. Finally, sparse envelope coefficients are extracted.

\section{Case Studies}

4.1. A Simulated Fault Signal Analyzed by the Proposed Method. In the first case study, a simulated signal is used for analyses. The simulated signal contains the impulses with an exponential decay, two sinusoidal signals, and noises. Here, the two sinusoidal signals can be regarded as two strong interruptions caused by two low-frequency components:

$$
\begin{aligned}
y(k)= & \sum_{r} \exp \left(-\alpha \times \frac{\left(k-r \times F_{s} / f_{m}-\tau_{r}\right)}{F_{s}}\right) \\
& \cdot \sin \left(2 \pi f_{1} \times \frac{\left(k-r \times F_{s} / f_{m}-\tau_{r}\right)}{F_{s}}\right) \\
& +0.9 \sin \left(\frac{2 \pi f_{2} k}{F_{s}}\right)+\sin \left(\frac{2 \pi f_{3} k}{F_{s}}\right)+n n(k),
\end{aligned}
$$

where $\alpha$ is equal to $900, n n(k)$ is the noise term, and $f_{m}$ is the modulating frequency (equal to $100 \mathrm{~Hz}$ ). $F_{s}$ is the sampling frequency set to $12000 \mathrm{~Hz}$. $f_{1}$ is the carrier frequency, equal to $3500 \mathrm{~Hz}$. $f_{2}$ and $f_{3}$ are sinusoidal frequencies, equal to $70 \mathrm{~Hz}$ and $140 \mathrm{~Hz}$, separately. 3600 samples are used. Normally distributed heavy noises with a mean of 0 and a variance of 0.2 are used as the noises. The simulated signal, the noise signal, and the mixed signal are shown in Figures 3(a), 3(b), and 3(c), respectively. From the mixed signal shown in Figure 3(c), it is hard to identify the periodic impulsive signal (the periodic intervals are equal to $10 \mathrm{~ms}$ ).
The proposed method is employed to analyze the mixed signal. First, the optimal parameters of the complex Morlet wavelet are automatically determined by using PSO with the maximum sparsity measurement. The optimal center frequency, the optimal bandwidth, and the frequency spectrum of the complex Morlet wavelet are shown in Figure 4(b). For a comparison, the frequency spectrum of the mixed signal is plotted in Figure 4(a). It is found that the optimal complex Morlet wavelet is correct to locate the simulated resonant frequency band around $3500 \mathrm{~Hz}$. Then, the mixed signal is filtered by the optimal complex Morlet wavelet and its corresponding frequency spectrum is given in Figure 4(c). The real part of the filtered signal is depicted in Figure 5(a), where it is seen that the in-band noises in the resonant frequency band still exist in the filtered signal. On the other hand, to validate the correction of the filtered signal, the power spectrum of the envelope of the filtered signal is plotted in Figure 5(b), where the fundamental frequency $100 \mathrm{~Hz}$ and its first two harmonics are identified. The global best values by using PSO are shown in Figure 6, where it is seen that sparsity value reaches the global optimal value quickly.

At last, the morphological analysis with the closing operator is employed to extract the cyclic bearing fault characteristics. The obtained temporal signal by morphological analysis is given in Figure $7(\mathrm{a})$. The absolute difference between the theoretical impulsive number and the actual impulsive number is shown in Figure 7(b), where the optimal length of 46 is found for morphological analysis. The local maximum locations of the signal shown in Figure $7(\mathrm{a})$ are displayed in Figure 7 (c). It is obvious that there is a pseudolocation. The iterative local maximum selection method is applied to process these local maximum locations shown in Figure 7(c). Finally, the revised local maximum locations are plotted in Figure 7(d). Compared with the signal shown in Figure 5(a), the result plotted in Figure 7(d) can be more understandable. Moreover, it is clear to see the signal having a cyclic interval of $10 \mathrm{~ms}$ by picking up the interval between two successive spikes. Besides, the final signal shown in Figure $7(d)$ is the sparse envelope coefficients of the simulated signal mixed with noise.

4.2. Experimental Fault Signals Analyzed by the Proposed Method. In this paper, the real motor bearing data picked up with a sampling frequency of $12 \mathrm{k} \mathrm{Hz}$ by an accelerometer at the drive end of the motor housing are used to validate the proposed method. A single-point defect was, respectively, introduced to the outer race and inner race of a normal bearing using electrodischarge machining. The fault diameter is 0.007 inches and the fault depth is 0.0011 inches. The motor load was $0 \mathrm{HP}$ and the motor speed was $1797 \mathrm{rpm}$ [33]. Outer race and inner race fault characteristic frequencies were calculated as $107 \mathrm{~Hz}$ and $162 \mathrm{~Hz}$, respectively.

The original outer race fault signal and its frequency spectrum are shown in Figures 8(a) and 8(b), respectively. The optimal parameters of the complex Morlet wavelet and its frequency spectrum are shown in Figure 8(c). Then, the optimal complex Morlet wavelet is used to retain one of the resonant frequency bands. The frequency spectrum of the signal obtained by the optimal wavelet filtering is displayed 


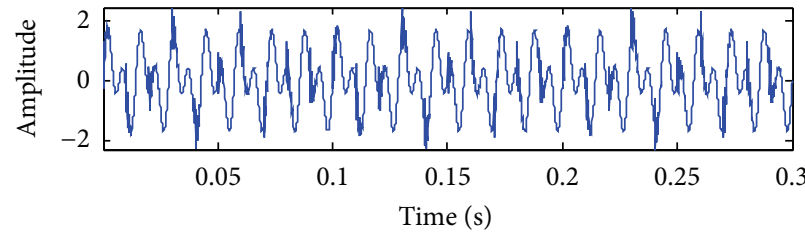

(a)

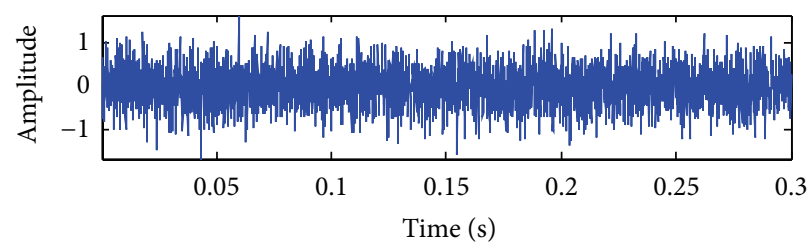

(b)

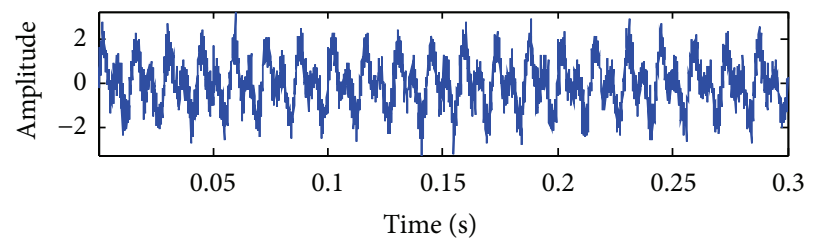

(c)

FIgURE 3: The simulated temporal signals: (a) the pure simulated signal consisting of impulses and two sinusoidal signals; (b) the noise signal; and (c) the pure simulated signal mixed with noises.

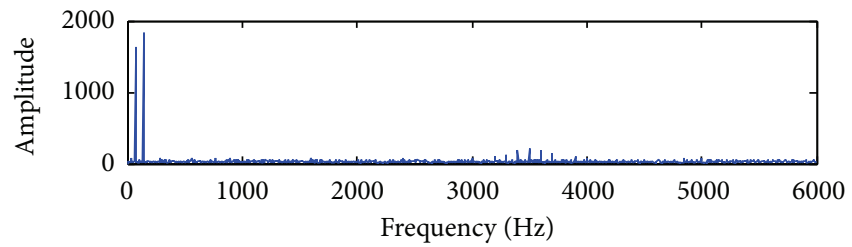

(a)

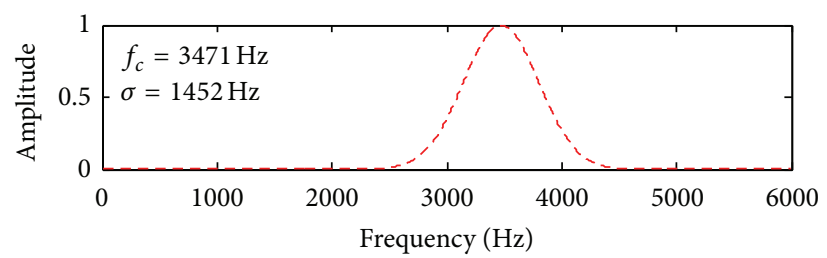

(b)

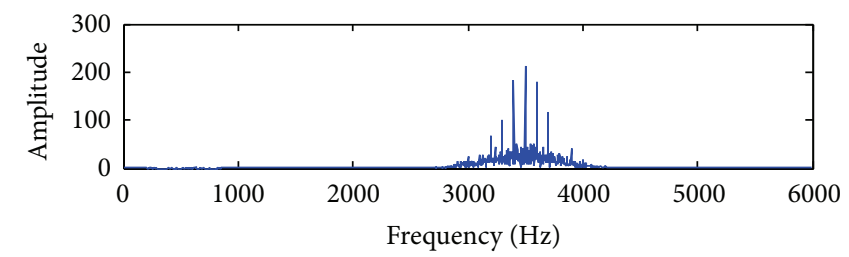

(c)

FIGURE 4: Frequency spectra: (a) the frequency spectrum of the mixed signal; (b) the frequency spectrum of the optimal complex Morlet wavelet; and (c) the frequency spectrum of the signal filtered by the optimal complex Morlet wavelet transform.

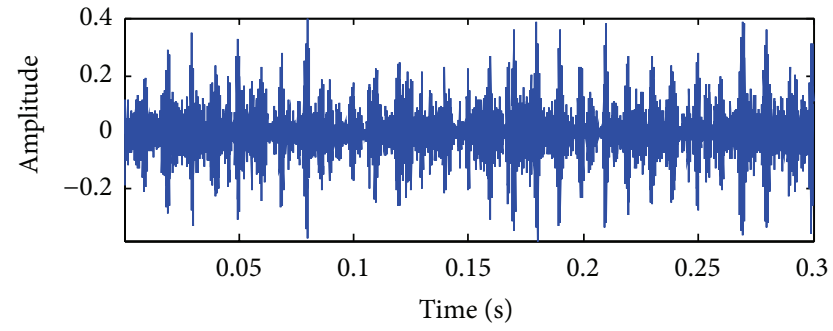

(a)

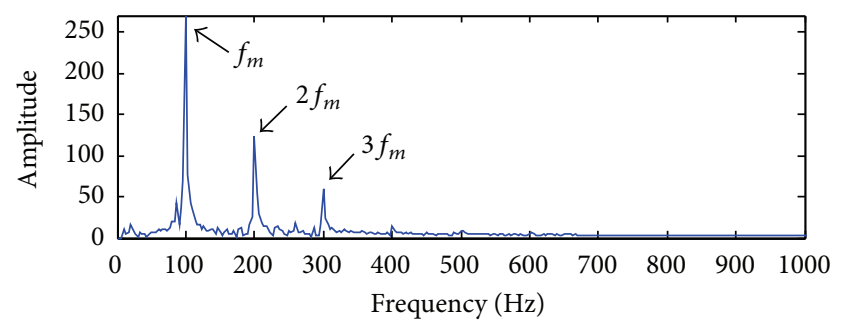

(b)

Figure 5: The signals obtained by the optimal complex Morlet filtering: (a) the real part of the filtered signal; (b) the power spectrum of the envelope of the filtered signal.

in Figure 8(d). It is clear to find that one of the resonant frequency bands is kept for further morphological analysis. The real part of the filtered signal and its envelope spectrum are shown in Figures 9(a) and 9(b), respectively, which demonstrate that the optimal filtering method correctly retains the major bearing fault signatures. The global best values by using particle swarm optimization are plotted in Figure 10. 


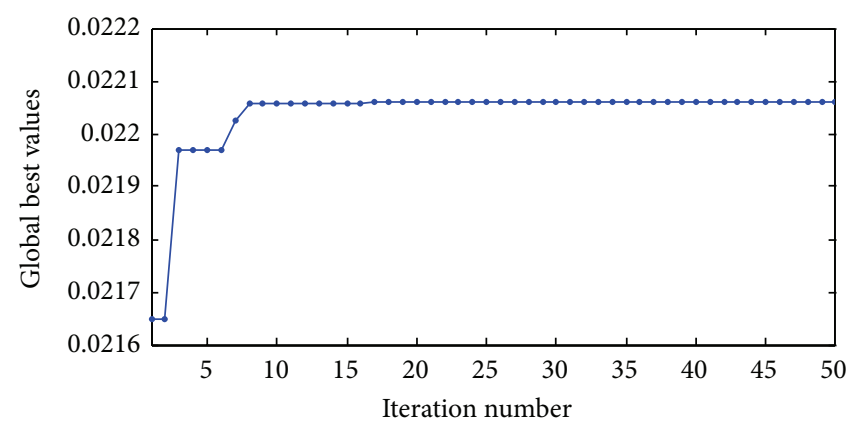

FIGURE 6: The global best values at different iterations by using PSO.

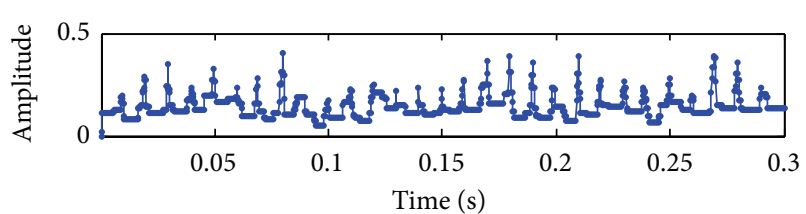

(a)

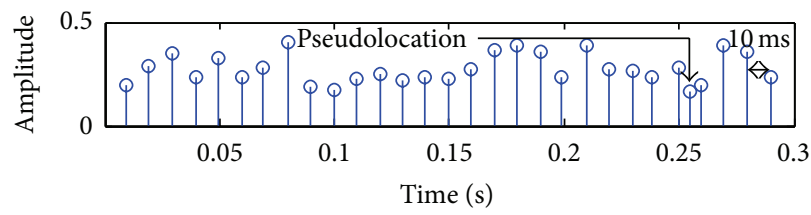

(c)

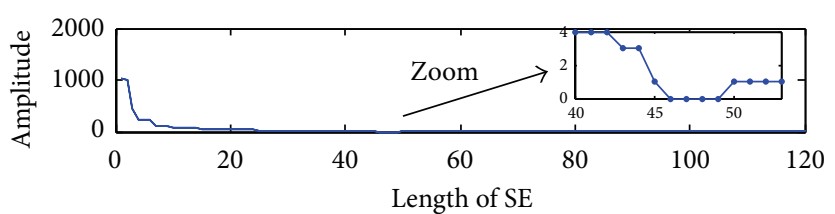

(b)

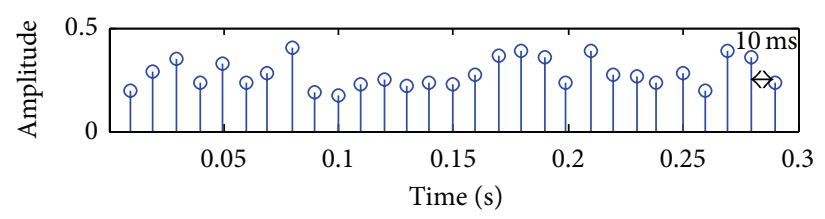

(d)

FIGURE 7: The results obtained by morphological analysis: (a) envelope extraction of the signal shown in Figure 5(a) by using the closing operator; (b) the optimal length of the SE; (c) the local maximum locations of the signal shown in Figure 7(a); and (d) the revised local maximum locations of the signal shown in Figure $7(\mathrm{c})$ by using the iterative local maximum selection.

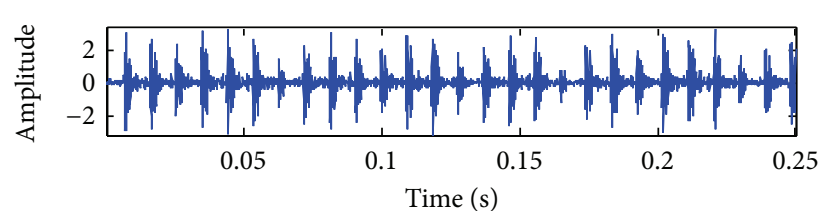

(a)

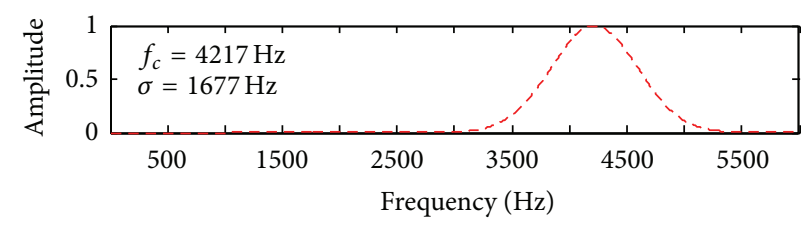

(c)

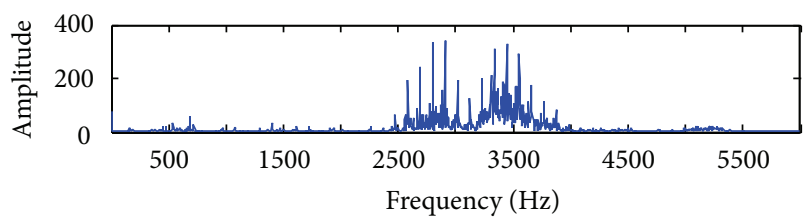

(b)

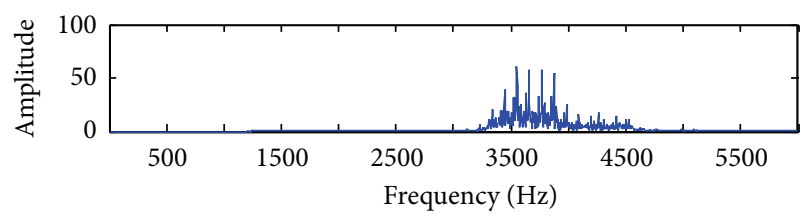

(d)

FIGURE 8: The signals: (a) the real outer race fault signal; (b) the frequency spectrum of the original outer race fault signal; (c) the frequency spectrum of the optimal complex Morlet wavelet; and (d) the frequency spectrum of the filtered signal.

Finally, morphological analysis is used to modify the shape of the signal shown in Figure 9(a). The extracted envelope by using the closing operator with the optimal length of the flat structuring element is displayed in Figure 11(a). The absolute differences are plotted in Figure 11(b) to find the optimal length of the flat structuring element. Here, the length of 29 is found. The local maximum locations of the envelope shown in Figure 11(a) are given in Figure 11(c).
It is obvious that the noises interrupt the local maximum locations. One pseudolocation is caused by an unexpected noise. Therefore, the iterative local maximum detection method is used to remove the irrelevant local maximum location. The result is shown in Figure 11(d). After removing the interruption caused by the unexpected noise, it is clear to see the pure cyclic fault characteristics with intervals of $9.3 \mathrm{~ms}$. 


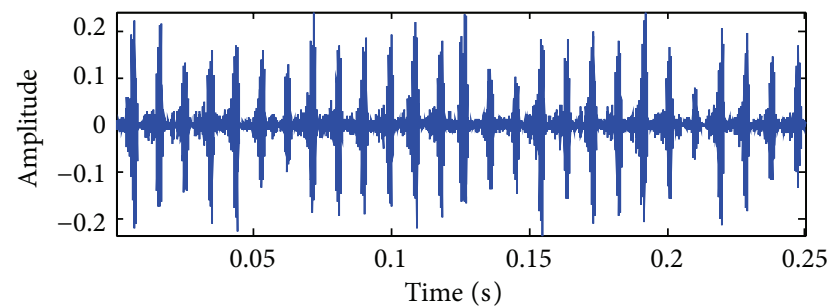

(a)

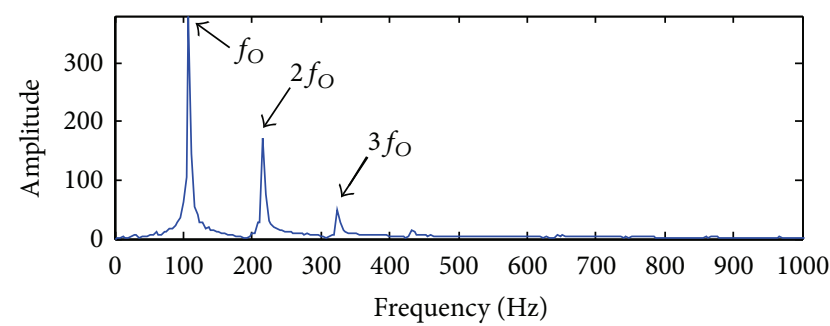

(b)

Figure 9: The signals obtained by the optimal complex Morlet filtering: (a) the real part of the filtered signal; (b) the power spectrum of envelope of the filtered signal.

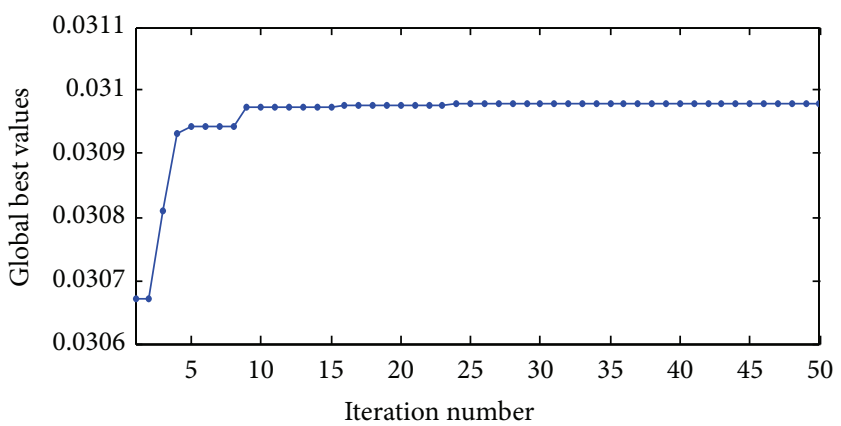

Figure 10: The global best values at different iterations by using PSO.

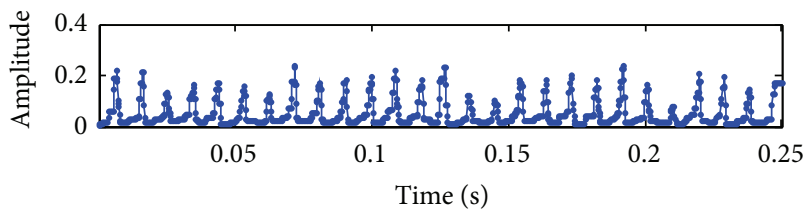

(a)

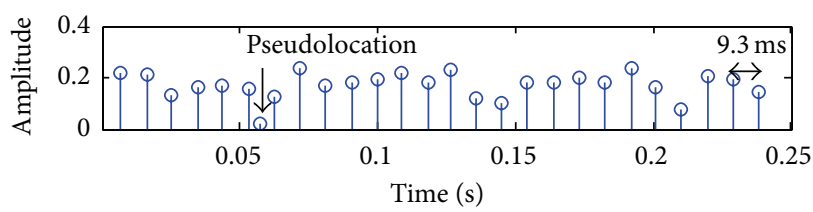

(c)

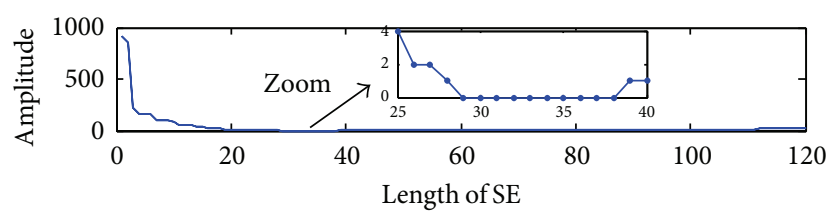

(b)

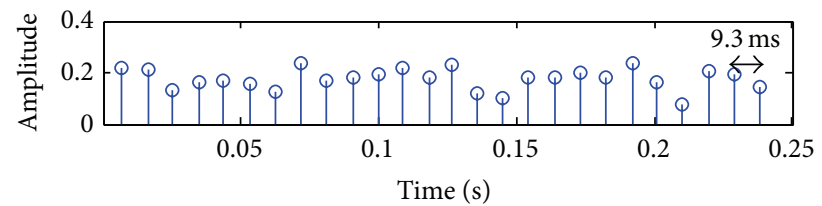

(d)

FIGURE 11: The results obtained by morphological filtering: (a) envelope extraction of the signal shown in Figure 9(a) by using the closing operator; (b) the optimal length of SE; (c) the local maximum locations of the signal shown in Figure 11(a); and (d) the revised local maximum locations of the signal shown in Figure 11(c) by using the iterative local maximum selection.

The same procedure is applied to process the inner race fault signal shown in Figure 12(a). The frequency spectrum of the inner race fault signal is plotted in Figure 12(b). The optimal parameters of the complex Morlet wavelet are given in Figure 12(c). The frequency spectrum of the filtered signal obtained by the optimal complex Morlet wavelet is displayed in Figure 12(d). Figures 13(a) and 13(b) show the real part of the filtered signal and its corresponding envelope spectrum. Obviously, the optimal complex Morlet wavelet transform reserves the inner race fault signatures. The global best values by using PSO are depicted in Figure 14.

The optimal process for the length selection of the flat structuring element is shown in Figure 15(b). The envelope obtained by using the closing operator with the optimal length is given in Figure 15(a). After that, the local maximum locations are extracted and the result is given in Figure 15(c). The pseudolocation is caused by an unexpected noise. In order to remove the irrelevant local maximum location shown in Figure 15(c), the iterative local maximum detection method is used. The final resulting signal is shown in Figure 15(d), where a pure cyclic fault signal is generated.

\section{Conclusion}

This paper reported a method which was used to extract sparse envelope coefficients for exhibiting bearing fault features. The proposed method consisted of two steps. Firstly, a Morlet wavelet was optimized by particle swarm optimization and then sparse wavelet coefficients were extracted from 


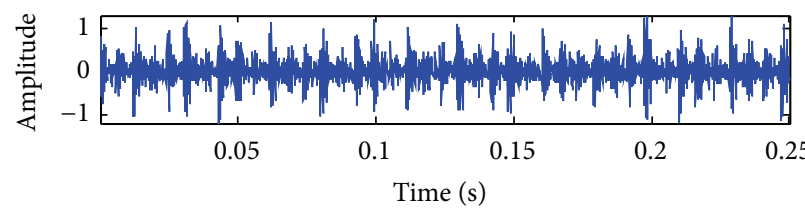

(a)

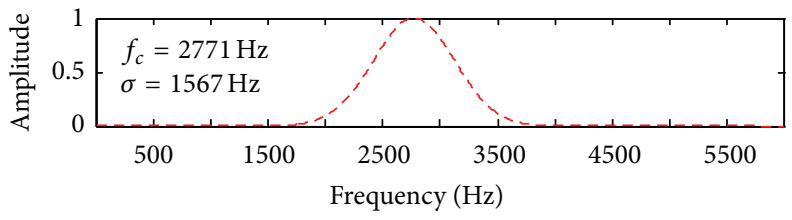

(c)

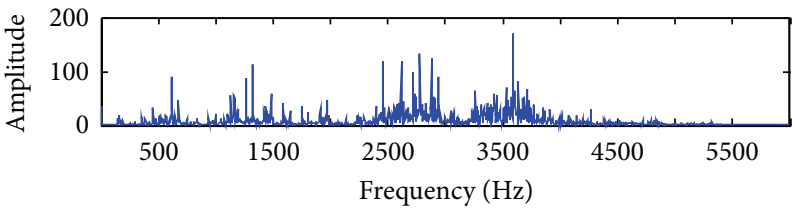

(b)

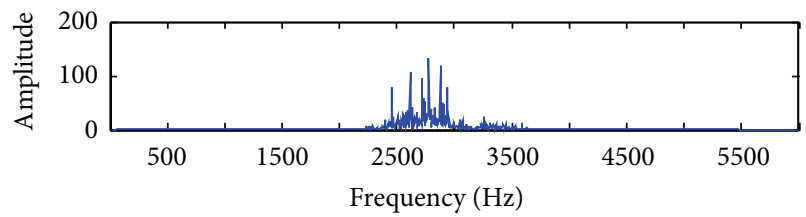

(d)

FIGURE 12: The signals: (a) the real inner race fault signal; (b) the frequency spectrum of the real inner race fault signal; (c) the frequency spectrum of the optimal complex Morlet wavelet; and (d) the frequency spectrum of the filtered signal.

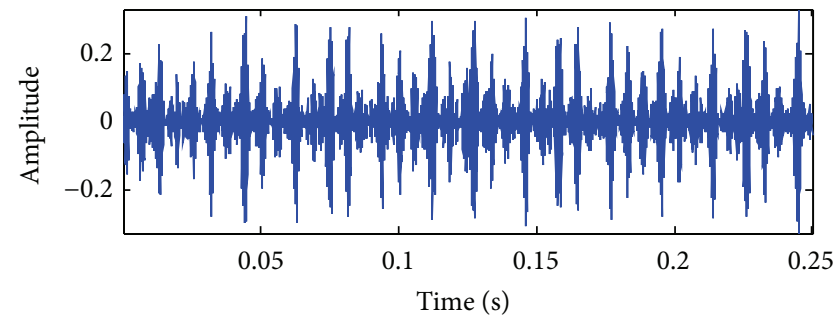

(a)

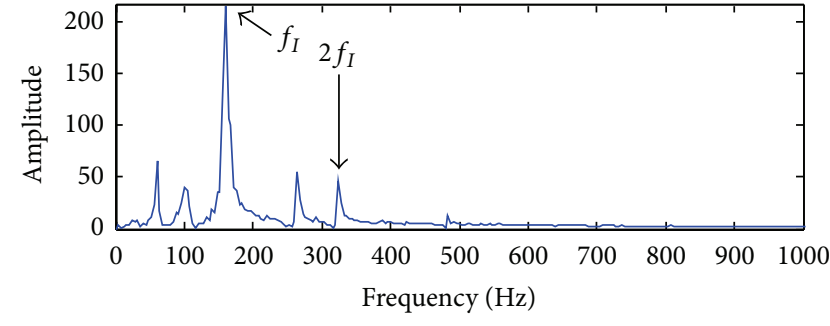

(b)

FIGURE 13: The signals obtained by the optimal complex Morlet filtering: (a) the real part of the signal filtered by the optimal Morlet wavelet transform; (b) the power spectrum of the envelope of the filtered signal.

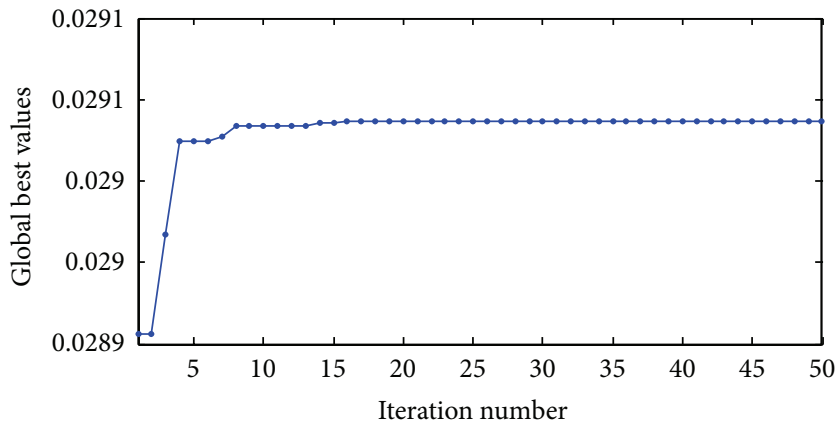

Figure 14: The global best values at every iteration by using particle swarm optimization.

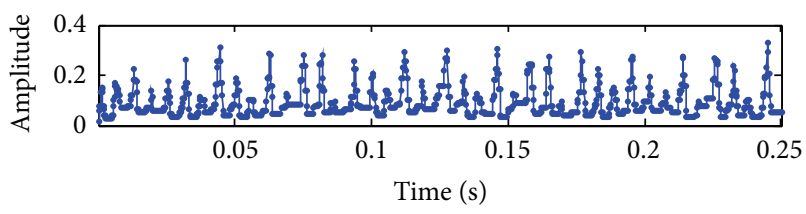

(a)

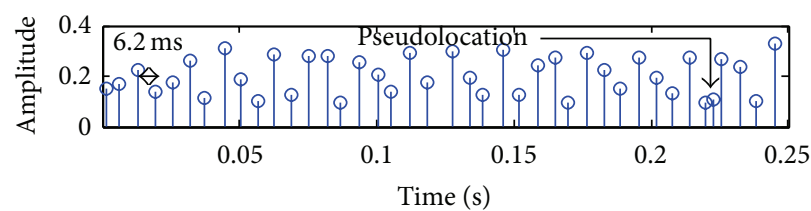

(c)

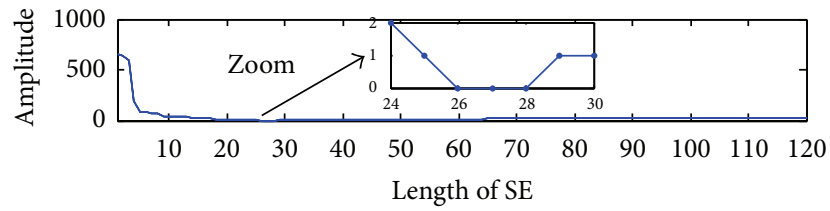

(b)

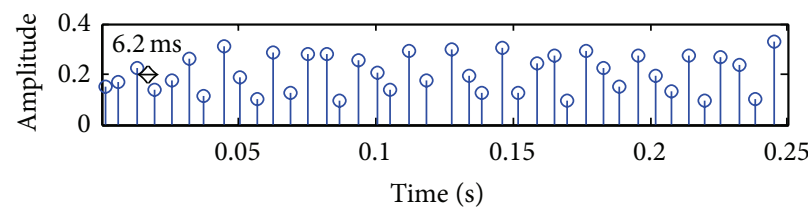

(d)

FIGURE 15: The results obtained by morphological filtering: (a) envelope extraction of the signal shown in Figure 13(a) by using the closing operator; (b) the optimal length of the flat SE; (c) the local maximum locations of the signal shown in (a); and (d) the revised local maximum locations of the signal shown in (c). 
bearing fault signals. Even though sparse wavelet coefficients were useful to represent bearing fault signatures, in-band noises still existed and could not be removed by using the solely optimal wavelet transform. In this step, the sparse wavelet coefficients were not succinct enough. Secondly, to reduce in-band noises, an adaptive morphological analysis with an iterative local maximum detection method was developed to postprocess the signal obtained by the optimal wavelet filtering. The optimal flat length used in morphological analysis with the closing operator was automatically determined to retain sparse envelope coefficients and remove the pseudo- and abnormal peaks caused by unexpected noises. After the above two steps were conducted, the sparse signal representation of bearing fault signals was obtained; only a few coefficients were used to represent bearing fault features. These sparse coefficients made bearing fault signals succinct and understandable. The case studies were conducted to illustrate how the proposed method worked and the results showed that the proposed method can be used to extract the sparse representation of bearing fault signals and identify different localized bearing faults.

In the future, the following works will be conducted. Firstly, more statistical metrics will be used as objective functions so as to guide optimization of wavelet transform. Secondly, besides the popular Morlet wavelet, more wavelets that are highly similar with impulses caused by bearing defects will be investigated. Thirdly, different optimization methods will be used to find optimal wavelet parameters and their comparisons will be made. Fourthly, even though wavelet transform is effective in extracting bearing fault features, in-band noises still exist. Morphological analysis with different structure elements and operators will be studied to make bearing fault features sparse and succinct. At last, based on bearing fault features extracted by the suggested steps, intelligent bearing fault diagnosis methods will be developed to automatically identify different bearing faults without requirement of expertise.

\section{Conflict of Interests}

The authors declare that there is no conflict of interests regarding the publication of this paper.

\section{Acknowledgments}

This research was partially supported by the National Natural Science Foundation of China (Grants nos. 51505311 and 51505307), the Science and Technology Foundation of Zhongshan City (Grant no. 20123A338), and the Natural Science Foundation of Jiangsu Province (Grant no. BK20150339). The authors would like to thank Professor K. A. Loparo for his permission to use the bearing data to validate the proposed method.

\section{References}

[1] S. Luo, J. Cheng, and H. Ao, "Application of LCD-SVD technique and CRO-SVM method to fault diagnosis for roller bearing," Shock and Vibration, vol. 2015, Article ID 847802, 8 pages, 2015.

[2] X. Zhang, J. Kang, L. Xiao, J. Zhao, and H. Teng, "A new improved kurtogram and its application to bearing fault diagnosis," Shock and Vibration, vol. 2015, Article ID 385412, 22 pages, 2015.

[3] Q. He, X. Ding, and Y. Pan, "Machine fault classification based on local discriminant bases and locality preserving projections," Mathematical Problems in Engineering, vol. 2014, Article ID 923424, 12 pages, 2014.

[4] Q. Miao, L. Cong, and M. Pecht, "Identification of multiple characteristic components with high accuracy and resolution using the zoom interpolated discrete Fourier transform," Measurement Science and Technology, vol. 22, no. 5, Article ID 055701, 2011.

[5] Y. Lei, J. Lin, Z. He, and Y. Zi, "Application of an improved kurtogram method for fault diagnosis of rolling element bearings," Mechanical Systems and Signal Processing, vol. 25, no. 5, pp. 1738-1749, 2011.

[6] D. Wang, P. W. Tse, and K. L. Tsui, "An enhanced Kurtogram method for fault diagnosis of rolling element bearings," Mechanical Systems and Signal Processing, vol. 35, no. 1-2, pp. 176-199, 2013.

[7] J. Antoni, "The spectral kurtosis: a useful tool for characterising non-stationary signals," Mechanical Systems and Signal Processing, vol. 20, no. 2, pp. 282-307, 2006.

[8] X. Chen, Z. Du, J. Li, X. Li, and H. Zhang, "Compressed sensing based on dictionary learning for extracting impulse components," Signal Processing, vol. 96, part A, pp. 94-109, 2014.

[9] W. He, Z.-N. Jiang, and K. Feng, "Bearing fault detection based on optimal wavelet filter and sparse code shrinkage," Measurement, vol. 42, no. 7, pp. 1092-1102, 2009.

[10] I. S. Bozchalooi and M. Liang, "A smoothness index-guided approach to wavelet parameter selection in signal de-noising and fault detection," Journal of Sound and Vibration, vol. 308, no. 1-2, pp. 246-267, 2007.

[11] W. Su, F. Wang, H. Zhu, Z. Zhang, and Z. Guo, "Rolling element bearing faults diagnosis based on optimal Morlet wavelet filter and autocorrelation enhancement," Mechanical Systems and Signal Processing, vol. 24, no. 5, pp. 1458-1472, 2010.

[12] H. Qiu, J. Lee, J. Lin, and G. Yu, "Robust performance degradation assessment methods for enhanced rolling element bearing prognostics," Advanced Engineering Informatics, vol. 17, no. 3-4, pp. 127-140, 2003.

[13] D. Wang, W. Guo, and X. Wang, "A joint sparse wavelet coefficient extraction and adaptive noise reduction method in recovery of weak bearing fault features from a multi-component signal mixture," Applied Soft Computing, vol. 13, no. 10, pp. 4097-4104, 2013.

[14] J. Lin and L. Qu, "Feature extraction based on morlet wavelet and its application for mechanical fault diagnosis," Journal of Sound and Vibration, vol. 234, no. 1, pp. 135-148, 2000.

[15] P. W. Tse and D. Wang, "The automatic selection of an optimal wavelet filter and its enhancement by the new sparsogram for bearing fault detection: part 2 of the two related manuscripts that have a joint title as 'Two automatic vibration-based fault diagnostic methods using the novel sparsity measurementparts 1 and 2"' Mechanical Systems and Signal Processing, vol. 40, no. 2, pp. 520-544, 2013.

[16] J. Kennedy and R. Eberhart, "Particle swarm optimization," in Proceedings of the IEEE International Conference on Neural Networks, vol. 4, pp. 1942-1948, IEEE, December 1995. 
[17] A. Banks, J. Vincent, and C. Anyakoha, "A review of particle swarm optimization. Part I: background and development," Natural Computing, vol. 6, no. 4, pp. 467-484, 2007.

[18] A. Banks, J. Vincent, and C. Anyakoha, "A review of particle swarm optimization. Part II: hybridisation, combinatorial, multicriteria and constrained optimization, and indicative applications," Natural Computing, vol. 7, no. 1, pp. 109-124, 2008.

[19] S.-F. Yuan and F.-L. Chu, "Fault diagnostics based on particle swarm optimisation and support vector machines," Mechanical Systems and Signal Processing, vol. 21, no. 4, pp. 1787-1798, 2007.

[20] B. Samanta and C. Nataraj, "Use of particle swarm optimization for machinery fault detection," Engineering Applications of Artificial Intelligence, vol. 22, no. 2, pp. 308-316, 2009.

[21] B. Samanta and C. Nataraj, "Application of particle swarm optimization and proximal support vector machines for fault detection," Swarm Intelligence, vol. 3, no. 4, pp. 303-325, 2009.

[22] N. G. Nlkolaou and I. A. Antoniadis, "Application of morphological operators as envelope extractors for impulsive-type periodic signals," Mechanical Systems and Signal Processing, vol. 17, no. 6, pp. 1147-1162, 2003.

[23] J. Wang, G. Xu, Q. Zhang, and L. Liang, "Application of improved morphological filter to the extraction of impulsive attenuation signals," Mechanical Systems and Signal Processing, vol. 23, no. 1, pp. 236-245, 2009.

[24] R. Hao, Z. Peng, Z. Feng, and F. Chu, "Application of support vector machine based on pattern spectrum entropy in fault diagnostics of rolling element bearings," Measurement Science and Technology, vol. 22, no. 4, Article ID 045708, 2011.

[25] B. Li, P.-L. Zhang, Z.-J. Wang, S.-S. Mi, and Y.-T. Zhang, "Gear fault detection using multi-scale morphological filters," Measurement, vol. 44, no. 10, pp. 2078-2089, 2011.

[26] B. Li, P.-L. Zhang, Z.-J. Wang, S.-S. Mi, and D.-S. Liu, "A weighted multi-scale morphological gradient filter for rolling element bearing fault detection," ISA Transactions, vol. 50, no. 4, pp. 599-608, 2011.

[27] C. Li, M. Liang, Y. Zhang, and S. Hou, "Multi-scale autocorrelation via morphological wavelet slices for rolling element bearing fault diagnosis," Mechanical Systems and Signal Processing, vol. 31, pp. 428-446, 2012.

[28] D. Wang, P. W. Tse, and Y. L. Tse, "A morphogram with the optimal selection of parameters used in morphological analysis for enhancing the ability in bearing fault diagnosis," Measurement Science and Technology, vol. 23, no. 6, Article ID 065001, 2012.

[29] C. Shen, Q. He, F. Kong, and P. W. Tse, "A fast and adaptive varying-scale morphological analysis method for rolling element bearing fault diagnosis," Proceedings of the Institution of Mechanical Engineers Part C: Journal of Mechanical Engineering Science, vol. 227, no. 6, pp. 1362-1370, 2013.

[30] S. Mallat, A Wavelet Tour of Signal Processing: the Sparse Way, Academic Press, Boston, Mass, USA, 2009.

[31] R. Yan, R. X. Gao, and X. Chen, "Wavelets for fault diagnosis of rotary machines: a review with applications," Signal Processing, vol. 96, pp. 1-15, 2014.

[32] L. Zhang, J. Xu, J. Yang, D. Yang, and D. Wang, "Multiscale morphology analysis and its application to fault diagnosis," Mechanical Systems and Signal Processing, vol. 22, no. 3, pp. 597610, 2008.

[33] X. Lou and K. A. Loparo, "Bearing fault diagnosis based on wavelet transform and fuzzy inference," Mechanical Systems and Signal Processing, vol. 18, no. 5, pp. 1077-1095, 2004. 


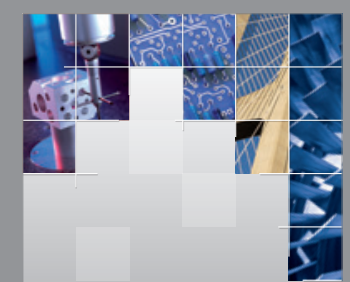

\section{Enfincering}
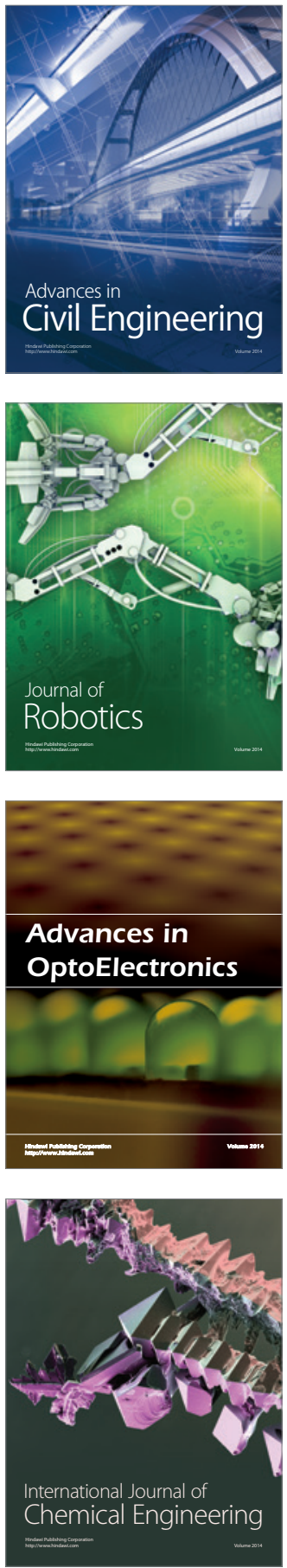

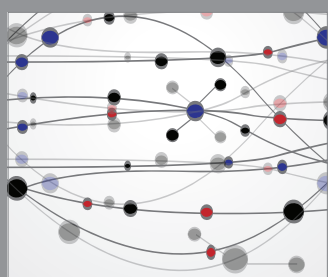

The Scientific World Journal

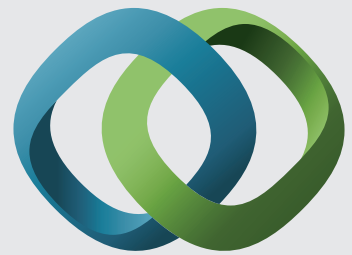

\section{Hindawi}

Submit your manuscripts at

http://www.hindawi.com
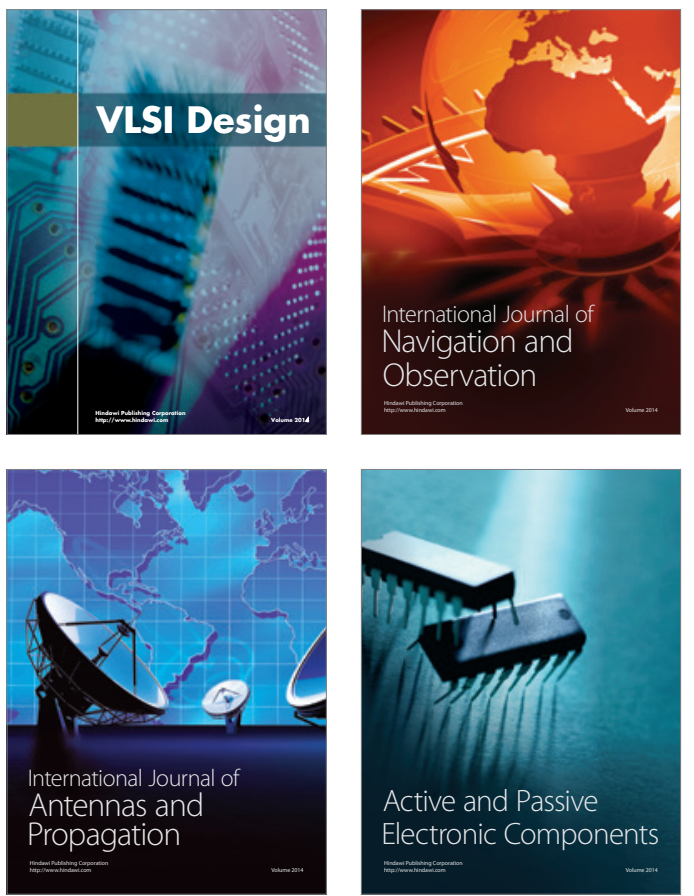
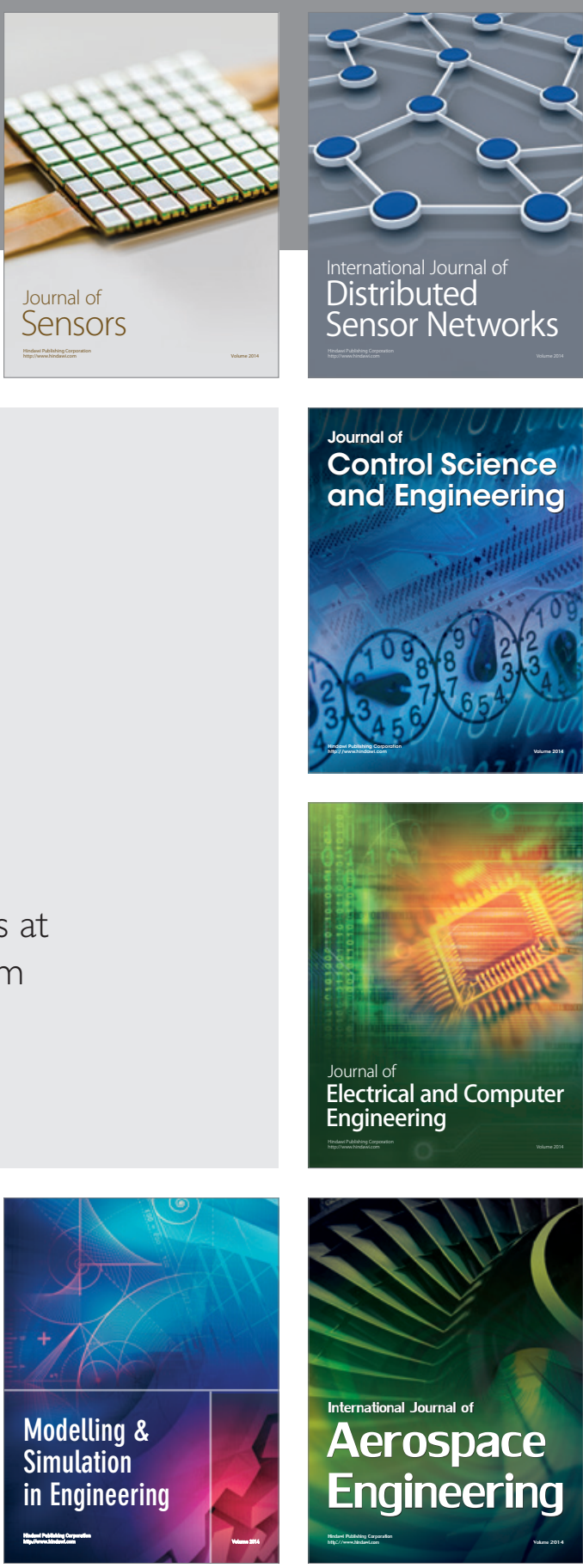

International Journal of

Distributed

Sensor Networks

Journal of

Control Science

and Engineering
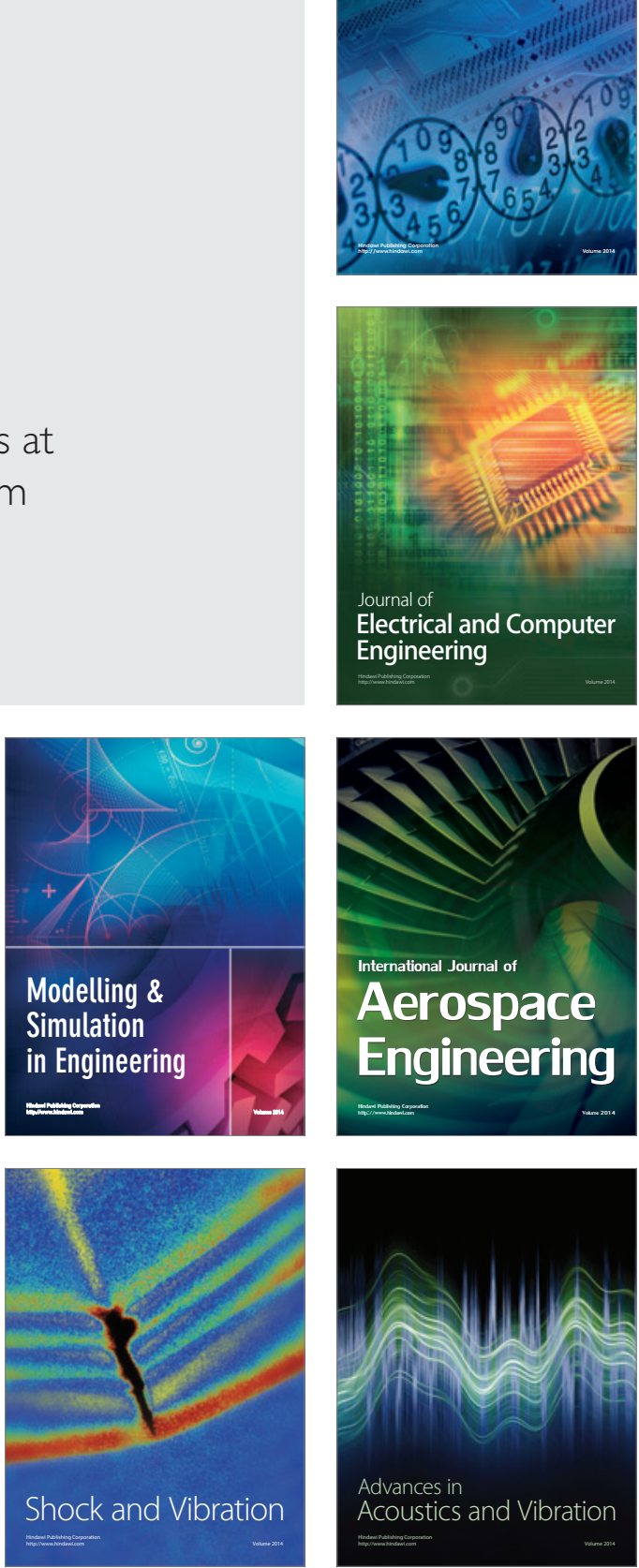